\title{
Pilot-Aided Acquisition Algorithms for Asynchronous DS-CDMA Systems*
}

\author{
Pei Xiao, ERIK StröM \\ Chalmers University of Technology, S-412 96 Göteborg, SWEDEN \\ pei.xiao@s2.chalmers.se,erik.strom@s2.chalmers.se
}

\begin{abstract}
The problem of estimating propagation delays of orthogonally modulated signals in asynchronous DSCDMA system over time-varing Rayleigh-fading channels is treated in this paper. The Maximum Likelihood (ML) estimator and its unaffordable complexity for implementation are discussed. Some suboptimal solutions, e.g., whitened sliding correlator, MMSE estimator, subspace-based estimator, approximate ML estimator are proposed to combat the multiple access interference in the fading channels. The performance of these estimators are evaluated with the computer simulations and shown to have better acquisition performance than the standard sliding correlator. They also achieve reduced computational complexity compared to the ML estimator, while maintaining an acceptable performance degradation.
\end{abstract}

\section{INTRODUCTION}

The considered system in this paper is a DS-CDMA system with orthogonal modulation. The transmitted chip sequence from a particular user is the concatenation of one of $M$ possible Walsh codes (representing the transmitted symbol) and a long scrambling (spreading) code used for user separation.

Accurate synchronization of the chip timing is required to achieve the low bit error rate. The impact of synchronization errors on demodulation of $M$-ary orthogonal signalling formats in DS-CDMA system was studied in [1]. It was shown that errors in the delay estimates would drastically degrade the system performance.

Synchronization issues were dealt with, e.g., in [2], [3], [4], etc.. However, the algorithms proposed in those papers only apply to CDMA systems with short spreading sequences and the modulation schemes other than orthogonal signalling. Long spreading codes have been included in several leading standard proposals for future cellular networks [5]. The idea of employing long codes is to randomize the interference and make them more noise-like. This will help systems combat the interference and some other channel impairments.

Synchronization of orthogonally modulated signals with long spreading sequences was explored in [6]. An adaptive algorithm was proposed to estimate the synchronization errors in synchronous CDMA systems. Based on

\footnotetext{
*This work has been partly presented at the European Wireless (EW) conference, Feb. 2002 and International Symposium on Spread Spectrum Techniques and Applications (ISSSTA), Sept. 2002.
}

the estimates, remedial actions are taken to alleviate the performance degradation caused by sampling the received signals at the incorrect timing. Simulation results show considerable capacity gains when the proposed algorithms are applied on erroneously sampled signals. The algorithms proposed in [6] are only applicable in the downlink scenario in which all the users transmit in a synchronous manner. On the uplink, different users transmit signals asynchronously, the propagation delays are therefore randomly distributed among different users. This paper aims at solving the problem of acquisition ${ }^{1}$ on the uplink.

In section 2, the system model is presented. Different acquisition schemes suitable for estimating propagation delays of the $M$-ary orthogonally modulated signals are introduced, their performance compared based on the numerical results in section 3. Conclusions are drawn in section 4 .

\section{System Model}

The passband received signal due to the $k^{t h}$ user is denoted by $r_{\mathrm{RF}, k}(t)$. The $k^{t h}$ user's $j^{t h}$ symbol is denoted by $i_{k}(j) \in\{1,2, \ldots, M\}$, and the $M$ orthogonal signal alternatives are defined by $M$ Walsh sequences $\left\{w_{1}(n), w_{2}(n), \ldots, w_{M}(n)\right\}$ of length $N$. The Walsh chips are randomized by a scrambling code $c_{k}(n) \in$ $\{-1,+1\}$. Hence, every symbol which represents $\log _{2} M$

\footnotetext{
${ }^{1}$ The term acquisition is used interchangeably with delay estimation when the propagation delay of the received signal is less than one symbol period $T$, which is assumed in this paper.
} 
binary bits is spread by $N$ chips and each bit by $N / \log _{2} M$ chips.

The baseband signal is formed by pulse amplitude modulation with the unit-energy rectangular chip waveform, and the baseband signal is multiplied with a carrier with frequency $\omega_{c}$ and transmitted over the channel, which is represented by the complex channel gain $h_{k}(t)$ and assumed to be a slowly time-varying Rayleigh flat fading channel with unknown propagation delay $\tau_{k} \in[0, T)$ ( $T=N T_{c}$ is the symbol duration, $T_{c}$ is the chip duration) and additive white Gaussian noise with double-sided power spectral density $N_{0} / 2$.

The total received signal is the sum of the $K$ users' signals plus additive white complex Gaussian noise $n(t)$. The complex envelope of the received signal is

$y(t)=n(t)+\sum_{k=1}^{K} y_{k}(t), y_{\mathrm{RF}, k}(t)=\sqrt{2} \operatorname{Re}\left\{y_{k}(t) e^{j \omega_{c} t}\right\}$

Let $r(t)$ denote the output from a filter matched to $\psi(t)$, i.e., $r(t)=y(t) * \psi(-t)$. We form the received vector, $\mathbf{r}(j) \in \mathbb{C}^{N}$, due to transmission of the $j^{\text {th }}$ symbol as

$$
\begin{aligned}
\mathbf{r}(j) & =\left[r\left([(j-1) N+1] T_{c}\right) \cdots r\left([(j-1) N+N] T_{c}\right)\right]^{T} \\
& =\mathbf{A}(j, \boldsymbol{\tau}) \mathbf{h}(j)+\mathbf{n}(j)
\end{aligned}
$$

The zero-mean complex Gaussian random vector $\mathbf{n}(j) \in \mathbb{C}^{N}$ has second moments ${ }^{2} \mathrm{E}\left[\mathbf{n}(i) \mathbf{n}^{T}(j)\right]=$ $\mathbf{0}$ and $\mathrm{E}\left[\mathbf{n}(i) \mathbf{n}^{*}(j)\right]=N_{0} \mathbf{I}_{N} \delta(i-j)$, where $\delta(\cdot)$ is the Kronecker delta function, The vector $\mathbf{h}(j) \in \mathbb{C}^{K}$ is defined by the complex channel gains as $\mathbf{h}(j)=$ $\left[h_{1}(j T) h_{2}(j T) \cdots h_{K}(j T)\right]^{T}$. The data matrix $\mathbf{A}(j, \boldsymbol{\tau}) \in$ $\mathbb{R}^{N \times K}$ can be expressed as

$$
\mathbf{A}(j, \boldsymbol{\tau})=\left[\begin{array}{llll}
\mathbf{a}_{1}\left(j, \tau_{1}\right) & \mathbf{a}_{2}\left(j, \tau_{2}\right) & \cdots & \mathbf{a}_{K}\left(j, \tau_{K}\right)
\end{array}\right]
$$

where $\tau_{k}$ stands for the propagation delay of the $k^{t h}$ user. The vector $\boldsymbol{\tau}$ is defined as $\boldsymbol{\tau}=\left[\tau_{1}, \tau_{2}, \ldots, \tau_{K}\right]^{T} \in \mathbb{R}^{N}$. Let us denote $\tau_{k}=\left(p_{k}+\delta_{k}\right) T_{c}$ ( $T_{c}$ is one chip interval) such that $p_{k} \in\{0,1, \ldots, N-1\}$ stands for the chip delay, $\delta_{k} \in[0,1)$ stands for the interchip delay, and denote $\mathbf{b}_{k}(j) \in \mathbb{R}^{N}$, the transmitted chip sequence due to the $k^{t h}$ user as

$$
\mathbf{b}_{k}(j)=\mathbf{C}_{k}(j) \mathbf{w}_{i_{k}(j)}
$$

where $\mathbf{C}_{k}(j) \in\{-1,+1\}^{N \times N}$ is a diagonal matrix defined by the $k^{t h}$ user's scrambling code, and $\mathbf{w}_{m} \in$ $\{-1,+1\}^{N \times 1}$ is the $m^{t h}$ column $\left(m=i_{k}(j)\right)$ of the $N \times N$ Hadamard matrix. For the rectangular pulse shape employed in this work, each column of the matrix $\mathbf{A}(j, \boldsymbol{\tau})$ in (2) can be expressed as

$$
\begin{aligned}
& \mathbf{a}_{k}\left(j, \tau_{k}\right)=\left(1-\delta_{k}\right)\left[\operatorname{ds}\left(\mathbf{b}_{k}(j), p_{k}\right)+\operatorname{us}\left(\mathbf{b}_{k}(j-1), N-p_{k}\right)\right] \\
& +\delta_{k}\left[\operatorname{ds}\left(\mathbf{b}_{k}(j), p_{k}+1\right)+\operatorname{us}\left(\mathbf{b}_{k}(j-1), N-p_{k}-1\right)\right]
\end{aligned}
$$

\footnotetext{
${ }^{2}$ the $N \times N$ identity matrix is denoted by $\mathbf{I}_{N}$. The transpose, conjugate transpose of a vector $\mathbf{x}$ are denoted by $\mathbf{x}^{T}, \mathbf{x}^{*}$, and $\|\mathbf{x}\|=\sqrt{\mathbf{x}^{*} \mathbf{x}}$, respectively.
}

where us(.), ds(.) stand for the up-shift and down-shift operators respectively:

$\operatorname{us}\left(\left[\begin{array}{lll}a_{1} & \cdots & a_{N}\end{array}\right]^{T}, q\right)=\left[\begin{array}{llllll}a_{N+1-q} & \cdots & a_{N} & 0 & \cdots & 0\end{array}\right]^{T}$, $\operatorname{ds}\left(\left[a_{1} \cdots \cdots a_{N}\right]^{T}, q\right)=\left[\begin{array}{lllllll}0 & \cdots & 0 & a_{1} & \cdots & a_{N-q}\end{array}\right]^{T}$.

For the periodic pilot signal employed for training at the delay estimation stage, $\mathbf{b}_{k}(j)$ remains unchanged. More will be said about the pilot sequence $\mathbf{b}_{k}(j)$ in the subsequent chapters when the acquisition algorithms are discussed.

\section{ACQUiSition Algorithms}

The delay estimation is the very first step to be conducted in the receiver. The task of the delay estimator in the receiver is to detect the propagation delays $\tau_{k}$ for $k=1,2, \ldots, K$ given the received signal vector $\mathbf{r}(j)$, i.e., perform acquisition jointly for all the users. For notation simplicity we sometimes suppress the symbol index $j$ from $\mathbf{a}_{k}\left(j, \tau_{k}\right), \mathbf{r}(j), \mathbf{h}(j), \mathbf{A}(j, \boldsymbol{\tau})$, etc., whenever no ambiguity arises.

The decision on the $k^{t h}$ user's delay $\tau_{k}$ is found as the maximizer/minimizer of the merit/cost function $J_{k}\left(\tau_{k}\right)$

$$
\hat{\tau}_{k}=\arg \max _{\tau_{k} \in[0, T)} / \min J_{k}\left(\tau_{k}\right)
$$

In this work, we define the failure of acquisition (estimation outlier) to be the cases when the estimated delay deviates from the true value in excess of half chip interval, i.e. $\left|\hat{\tau}_{k}-\tau_{k}\right|>T_{c} / 2$.

In the following, we shall introduce how the decision function $J_{k}\left(\tau_{k}\right)$ is derived for different acquisition algorithms.

\subsection{Approach to Delay Estimation}

In our case, the noise is complex Gaussian. Given $L$ observations of the received vector $\mathbf{r}(j)$ for $j=1,2, \ldots, L$, the log-likelihood function of the received vector conditioned on a realization of the fading channels and propagation delays can be expressed as

constant $-L N \ln \left(N_{0} / 2\right)-\frac{2}{N_{0}} \sum_{j=1}^{L}\|\mathbf{r}(j)-\mathbf{A}(j, \boldsymbol{\tau}) \mathbf{h}(j)\|^{2}$

From (5), one can see that maximization of this loglikelihood function is equivalent to minimization of the function

$$
\sum_{j=1}^{L}\|\mathbf{r}(j)-\mathbf{A}(j, \boldsymbol{\tau}) \mathbf{h}(j)\|^{2}
$$

It is shown in [7] that the ML estimation of the fading channel vector $\mathbf{h}(j)$ is $\hat{\mathbf{h}}(j)=\mathbf{A}^{\dagger} \mathbf{r}(j)$ Substituting $\hat{\mathbf{h}}(j)$ into (6), we yield the ML estimate of $\boldsymbol{\tau}=$ 


$$
\begin{aligned}
& {\left[\tau_{1}, \tau_{2}, \ldots, \tau_{K}\right]^{T} \text { as }} \\
& \hat{\boldsymbol{\tau}}^{\mathrm{ML}}=\left.\arg \min _{\boldsymbol{\tau}} \sum_{j=1}^{L}\|\mathbf{r}(j)-\mathbf{A}(j, \boldsymbol{\tau}) \mathbf{h}(j)\|^{2}\right|_{\hat{\mathbf{h}}(j)=\mathbf{A}^{\dagger} \mathbf{r}(j)} \\
& \quad=\arg \min _{\boldsymbol{\tau}} \operatorname{trace}\left(\mathbf{P}_{\mathbf{A}}^{\perp} \hat{\mathbf{R}}\right)
\end{aligned}
$$

where $\mathbf{P}_{\mathbf{A}}^{\perp}=\mathbf{I}_{N}-\mathbf{A} \mathbf{A}^{\dagger}$ is the orthogonal projection matrix onto the orthogonal complement to the subspace spanned by the columns of $\mathbf{A}(j, \boldsymbol{\tau})$ and $\hat{\mathbf{R}}$ is the sample covariance matrix defined by $\hat{\mathbf{R}}=\frac{1}{L} \sum_{j=1}^{L} \mathbf{r}(j) \mathbf{r}^{*}(j)$.

The ML delay estimator finds the delays of all the users simultaneously. The disadvantage of this algorithm is the unaffordable computational complexity because it has to search over $N^{K}$ points ( $K$ is the number of users, $N$ is spreading factor, and we assume the delays are within one symbol interval). While other estimators introduced below estimate the delays for one user at a time, therefore, the number of points to be searched goes down to $N K$. The sub-optimum solutions with reduced complexity are discussed below.

\subsection{Conventional Sliding Correlator}

The sliding correlator (SC) is the standard approach to propagation delay estimation. It treats the multiple access interference (MAI) as additive noise. The received signal is correlated with time delayed versions of the training sequence, and the desired timing is the value of the time delay candidate that maximizes the correlation. Mathematically, this estimate is given by

$$
\begin{aligned}
\hat{\tau}_{k}^{c} & =\arg \max _{\tau_{k} \in[0, T)} J_{k, C}\left(\tau_{k}\right) \\
& =\arg \max _{\tau_{k} \in[0, T)} \frac{\left|\hat{\mathbf{a}}_{k}\left(\tau_{k}\right)^{*} \overline{\mathbf{r}}\right|^{2}}{\left\|\hat{\mathbf{a}}_{k}\left(\tau_{k}\right)\right\|^{2}}
\end{aligned}
$$

where $\overline{\mathbf{r}}=\frac{1}{L} \sum_{j=1}^{L} \mathbf{r}(j)$ is the sample mean.

The advantages of the SC are the low computational complexity and good performance in single user situation. However, it is highly unreliable in presence of the MAI, even in two-user case, as demonstrated in figure 1 (In this paper, all the performance results are obtained with computer simulations. 'Percentage of outliers' is used interchangeably with 'probability of acquisition failure').

This approach as well as other acquisition schemes introduced in the following subsections require a training sequence which is periodic for each user and with good crosscorrelation property among the users. This can be fulfilled by assigning a distinct Walsh sequence to each user and spreading it with a short scrambling code to achieve low cross-correlation between the shifted versions of Walsh sequences, then transmitting the same scrambled symbols repeatedly.

The simulated system for this and all the following experiments is a $K=6$-user system with $N=32$ chips

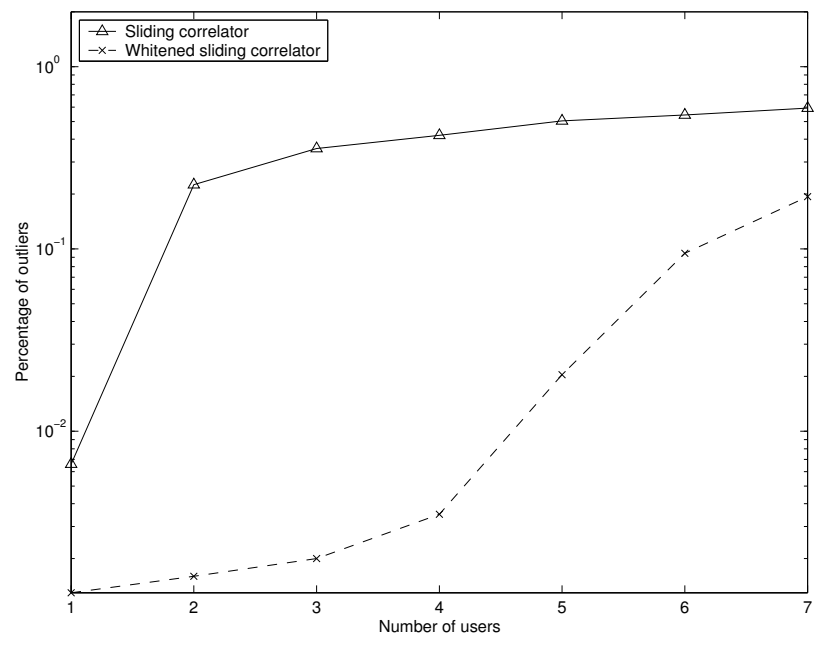

Figure 1: Performance of SC and WSC.

per symbol and $M=8$ signal alternatives. The scrambling codes $c_{k}(n)$ are random and also periodic (remain the same during each symbol period). Perfect power control is assumed. The signal to noise ratio is set to $10 \log _{10} E_{b} / N_{0}=$ $20 d B$, except in the SNR test. The channels are independent Rayleigh fading channels. The channel gain $h_{k}(t)$ is complex circular Gaussian process with autocorrelation function $\mathrm{E}\left[h_{k}^{*}(t) h_{k}(t+\tau)\right]=J_{0}\left(2 \pi f_{D} \tau\right)$ where $f_{D}$ is the maximum Doppler frequency and $J_{0}(x)$ is the zeroth order Bessel function of the first kind. The normalized Doppler frequency is $f_{D} T=0.01$, which represents rather fast fading channels, but slow enough for the channel gain to remain approximately constant during one symbol duration. Simulation results are averaged over $500 \sim 3000$ MonteCarlo runs with $t=10 \sim 300$ pilot symbols transmitted. Each MCrun represents a particular realization of the noise and fading processes as well as randomly distributed propagation delays $\tau_{k} \in[0, T)$.

\subsection{Whitened SLiding Correlator}

The conventional sliding correlator achieves good performance in the AWGN single user channel or in strict orthogonal synchronous channel. It performs poorly when the white Gaussian noise is incorporated with the MAI. A way to work around this problem is to whiten the received vector $\mathbf{r}$ by preprocessing it with the matrix $\mathbf{R}^{-1 / 2}$ [2].

The received vector can be written as

$$
\mathbf{r}=h_{k} \mathbf{a}_{k}+\underbrace{\sum_{\substack{l=1 \\ l \neq k}}^{K} h_{l} \mathbf{a}_{l}}_{\text {MAI }}+\mathbf{n}
$$

The "whitened" received vector is

$$
\mathbf{R}^{-1 / 2} \mathbf{r}=\mathbf{R}^{-1 / 2}(\mathbf{A} \mathbf{h}+\mathbf{n})=\underbrace{h_{k} \mathbf{R}^{-1 / 2} \mathbf{a}_{k}}_{\text {desired signal }}+\underbrace{\mathbf{R}^{-1 / 2}(\mathrm{MAI}+\mathbf{n})}_{\text {noise }}
$$


where $\mathbf{R}^{-1 / 2}$ is obtained from $\mathbf{R}$ by Cholesky factorization, it exists as long as $\mathbf{R}$ is positive definite, which is the case if $N_{0}>0$. The matrix $\mathbf{R}$ is the covariance matrix for $\mathbf{r}$, and can be calculated as

$$
\mathbf{R}=\mathrm{E}\left[\mathbf{r r}^{*}\right]=\mathbf{A} \mathrm{E}\left[\mathbf{h h}^{*}\right] \mathbf{A}^{*}+N_{0} \mathbf{I}_{N}=\mathbf{A} \mathbf{P} \mathbf{A}^{*}+N_{0} \mathbf{I}_{N}
$$

The enhanced version of the SC, namely, the whitened sliding correlator (WSC) can be formulated as

$$
J_{k, W}\left(\tau_{k}\right)=\frac{\left|\left(\hat{\mathbf{R}}^{-1 / 2} \mathbf{a}_{k}\left(\tau_{k}\right)\right)^{*} \hat{\mathbf{R}}^{-1 / 2} \overline{\mathbf{r}}\right|}{\left\|\hat{\mathbf{R}}^{-1 / 2} \mathbf{a}_{k}\left(\tau_{k}\right)\right\|^{2}}=\frac{\left|\mathbf{a}_{k}\left(\tau_{k}\right)^{*} \hat{\mathbf{R}}^{-1} \overline{\mathbf{r}}\right|}{\mathbf{a}_{k}\left(\tau_{k}\right)^{*} \hat{\mathbf{R}}^{-1} \mathbf{a}_{k}\left(\tau_{k}\right.}
$$

The covariance matrix $\mathbf{R}$ is unknown and usually replaced by the sample covariance matrix $\hat{\mathbf{R}}$.

As seen from (4), $\mathbf{a}_{k}\left(\tau_{k}\right)$ is piecewise linear in $\tau_{k}$. In particular, for $\tau_{k} \in\left[p T_{c},(p+1) T_{c}\right)$

$$
\mathbf{a}_{k}\left(\tau_{k}\right)=\delta \mathbf{a}_{k}\left(t_{1}\right)+(1-\delta) \mathbf{a}_{k}\left(t_{0}\right)
$$

where $t_{0}=p T_{c}, t_{1}=(p+1) T_{c}, p$ is an integer and $\delta=$ $\left(\tau_{k}-t_{0}\right) / T_{c}$. By combining (11) and (12), we obtain

$$
\begin{aligned}
\hat{\tau}_{k}^{w} & =\arg \max _{\tau_{k} \in[0, T)} J_{k, W}^{2}\left(\tau_{k}\right) \\
& =\arg \max _{\tau_{k} \in[0, T)} \frac{\left\|K_{2}-K_{1}\right\|^{2} \delta^{2}+X_{0} \delta+\left\|K_{1}\right\|^{2}}{X_{1} \delta^{4}+X_{2} \delta^{3}+X_{3} \delta^{2}+X_{4} \delta+K_{3}^{2}}
\end{aligned}
$$

where $K_{1}=\mathbf{a}_{k}\left(t_{0}\right)^{*} \mathbf{R}^{-1} \mathbf{r}, K_{2}=\mathbf{a}_{k}\left(t_{1}\right)^{*} \mathbf{R}^{-1} \mathbf{r}, K_{3}=$ $\mathbf{a}_{k}\left(t_{0}\right)^{*} \mathbf{R}^{-1} \mathbf{a}_{k}\left(t_{0}\right), K_{4}=\mathbf{a}_{k}\left(t_{1}\right)^{*} \mathbf{R}^{-1} \mathbf{a}_{k}\left(t_{1}\right), K_{5}=$ $\operatorname{Re}\left\{\mathbf{a}_{k}\left(t_{0}\right)^{*} \mathbf{R}^{-1} \mathbf{a}_{k}\left(t_{1}\right)\right\}, X_{0}=\left[K_{1}^{*}\left(K_{2}-K_{1}\right)+K_{1}\left(K_{2}-\right.\right.$ $\left.\left.K_{1}\right)^{*}\right], X_{1}=\left(K_{3}+K_{4}-2 K_{5}\right)^{2}, X_{2}=4\left(K_{3}+K_{4}-\right.$ $\left.2 K_{5}\right)\left(K_{5}-K_{3}\right), X_{3}=2\left(K_{3}+K_{4}-2 K_{5}\right) K_{3}, X_{4}=$ $4\left(K_{5}-K_{3}\right) K_{3}$.

Note that we use $J_{k, W}^{2}$ to replace $J_{k, W}$ of equation (11) in order to make the numerator differentiable.

From (13) we see that $J_{k, W}^{2}\left(\tau_{k}\right)$ can be written as a rational function of two polynomials of degree six. Furthermore, $J_{k, W}^{2}\left(\tau_{k}\right)$ is differentiable for $\tau_{k} \in[0, T)$ except at the points of $\tau_{k}=p T_{c}$ for an integer $p$. This suggests that we can search the set of candidate estimates $\mathcal{T}$, of the delay $\tau_{k}$ in all the $N$ bins $\tau_{k} \in\left(p T_{c},(p+1) T_{c}\right)$ for $p=0,1, \ldots, N-1$ as the solutions to $\frac{d J_{k, W}^{2}\left(\tau_{k}\right)}{d \tau_{k}}=0$, as well as those end points $p T_{c}$. The final estimates, $\hat{\tau}_{k}$, is found as the member of $\mathcal{T}$ corresponding to the largest value of the merit function $J_{k, W}^{2}\left(\tau_{k}\right)$.

Figure 1 shows that the acquisition performance of the WSC is greatly improved in presence of the MAI compared to the conventional SC.

With the WSC and all the algorithms introduced later on, each user has to transmit the same pilot symbol during the training period so that the data matrix $\mathbf{A}$ in equation (10) remains unchanged. The number of pilot symbols needs to be sufficiently large in order to get an accurate estimate of $\mathbf{R}$. Figure 2 shows that the acquisition

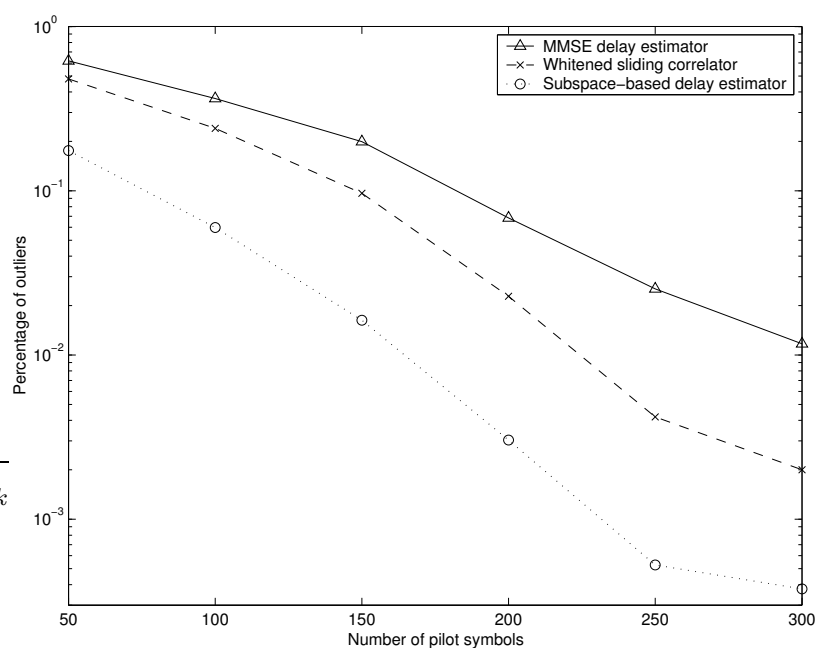

Figure 2: Acquisition error probability as a function of the number of training symbols.

error probability decreases when more pilot symbols are used. Long training sequences are needed to combat fading. The reason is that the covariance of the fading channel vector, after averaging, approximates the matrix $\mathbf{P}$, i.e., $\frac{1}{L} \sum_{j=1}^{L} \mathbf{h}(j) \mathbf{h}^{*}(j) \cong \mathbf{P}=\operatorname{diag}\left(P_{1}, P_{2}, \ldots, P_{K}\right)$, which leads to $\frac{1}{L} \sum_{j=1}^{L} \mathbf{r}(j) \mathbf{r}^{*}(j) \cong \mathbf{R}$. The same analysis holds for other algorithms, as indicated in figure 2 .

\subsection{LINEAR MMSE DELAY ESTIMATOR}

The linear MMSE delay estimation is based on the MMSE approach to data detection [3]. The receiver computes a receiver matrix $\mathbf{W} \in C^{N \times N}$, which is chosen to minimize the mean square error $\mathrm{E}\left[\left\|\mathbf{a}_{k}\left(\tau_{k}\right) h_{k}-\mathbf{W}^{*} \mathbf{r}\right\|^{2}\right]$. The receiver matrix can be computed using adaptive filtering technique

$$
\begin{aligned}
\mathbf{W}_{m m s e} & =\arg \min _{\mathbf{W}} \mathrm{E}\left[\left\|\mathbf{a}_{k}\left(\tau_{k}\right) h_{k}-\mathbf{W}^{*} \mathbf{r}\right\|^{2}\right]=\mathbf{R}^{-1} \mathbf{U} \\
\mathbf{U} & =\mathrm{E}\left[\mathbf{r}\left(\mathbf{a}_{k}\left(\tau_{k}\right) h_{k}\right)^{*}\right]=\mathrm{E}\left[\mathbf{a}_{k}\left(\tau_{k}\right) h_{k} h_{k}^{*} \mathbf{a}_{k}^{*}\left(\tau_{k}\right)\right] \\
& =\mathbf{a}_{k}\left(\tau_{k}\right) \mathbf{a}_{k}^{*}\left(\tau_{k}\right)
\end{aligned}
$$

If we look into the received vector $\mathbf{r}$ in (9), the contribution from the $k^{t h}$ user is $h_{k} \mathbf{a}_{k}$. This implies the rationale behind the equation (14) is that we would like to find a matrix $\mathbf{W}$ which can filter out all the interference and noise from the received observation so that in the ideal situation, the received vector after filtering only contains the $k^{t h}$ user's contribution. Once the receiver matrix is computed, the delay can be estimated by correlating this matrix with the received vector. Thus, the MMSE approach to de- 
lay estimation can be formulated as

$$
\begin{aligned}
\hat{\tau}_{k}^{M M S E} & =\arg \max _{\tau_{k} \in[0, T)} J_{k, M M S E}\left(\tau_{k}\right) \\
& =\arg \max _{\tau_{k} \in[0, T)} \frac{\left\|\mathbf{W}_{m m s e}^{*} \overline{\mathbf{r}}\right\|^{2}}{\left\|\mathbf{W}_{m m s e}\right\|^{2}} \\
& =\arg \max _{\tau_{k} \in[0, T)} \frac{\left\|\left(\hat{\mathbf{R}}^{-1} \hat{\mathbf{a}}\left(\tau_{k}\right) \hat{\mathbf{a}}\left(\tau_{k}\right)^{*}\right)^{*} \overline{\mathbf{r}}\right\|^{2}}{\left\|\hat{\mathbf{R}}^{-1} \hat{\mathbf{a}}\left(\tau_{k}\right) \hat{\mathbf{a}}\left(\tau_{k}\right)^{*}\right\|^{2}}
\end{aligned}
$$

where $\overline{\mathbf{r}}$ and $\hat{\mathbf{R}}$ are the sample mean of the received vector and covariance matrix respectively. The analytical expression of $J_{k, M M S E}$ is omitted to conserve space.

As depicted in figure 2, the performance of the MMSE estimator is close to that of the WSC when the number of pilots is small. The gap becomes bigger when the number of pilots increases.

\subsection{SubSPACE-BASEd DELAY ESTIMATOR}

The subspace-based approach to delay estimation was initially proposed in [4], [8]. We assume that $\mathbf{A}$ and $\mathbf{P}$ in the equation (10) have full rank ${ }^{3}$ and define the signal subspace as the column space of $\mathbf{A}$, i.e., range $(\mathbf{A})$, and the noise subspace as the orthogonal complement to range $(\mathbf{A})$. It can be shown that range $\left(\mathbf{A P A} \mathbf{A}^{*}\right)=$ range(A). Furthermore, since $\mathbf{A}$ has rank $K$ (the number of users), the signal subspace will have dimensionality $K$. Since $\mathbf{R}$ is hermitian and positive definite, there exist an eigenvalue decomposition of $\mathbf{R}$ as

$$
\mathbf{R}=\mathbf{E}_{s} \Lambda_{s} \mathbf{E}_{s}^{*}+N_{0} \mathbf{E}_{n} \mathbf{E}_{n}^{*}
$$

where $\mathbf{E}_{s} \in \mathbb{C}^{N \times K}$ and $\mathbf{E}_{n} \in \mathbb{C}^{N \times(N-K)}$ are such that $\left[\mathbf{E}_{s} \mathbf{E}_{n}\right]$ is unitary, and $\Lambda_{s} \in \mathbb{R}^{K \times K}$ is defined as

$$
\Lambda_{s}=\operatorname{diag}\left(\lambda_{1}+N_{0}, \lambda_{2}+N_{0}, \ldots, \lambda_{K}+N_{0}\right)
$$

where $\left\{\lambda_{1}, \ldots, \lambda_{K}\right\}$ are the non-zero eigenvalues of APA*.

Now we note that $\operatorname{range}(\mathbf{A})=\operatorname{range}\left(\mathbf{E}_{s}\right)$. Consequently, the columns of $\mathbf{A}\left(\mathbf{a}_{1}\left(\tau_{1}\right), \mathbf{a}_{2}\left(\tau_{2}\right), \ldots, \mathbf{a}_{K}\left(\tau_{K}\right)\right)$ are orthogonal to the columns of $\mathbf{E}_{n}$. Given knowledge of $\mathbf{R}$ we can find $\tau_{k}$ as the solutions to $\left\|\mathbf{E}_{n}^{*} \mathbf{a}_{k}\left(\tau_{k}\right)\right\|^{2}=0$. In practice, the covariance matrix is unknown and is therefore estimated by the sample covariance matrix $\hat{\mathbf{R}}$, and a consistent estimate of $\mathbf{E}_{n}$ is found in the eigenvalue decomposition of $\hat{\mathbf{R}}$

$$
\hat{\mathbf{R}}=\frac{1}{L} \sum_{l=1}^{L} \mathbf{r}(l) \mathbf{r}^{*}(l)=\hat{\mathbf{E}}_{s} \hat{\Lambda}_{s} \hat{\mathbf{E}}_{s}^{*}+\hat{\mathbf{E}}_{n} \hat{\Lambda}_{n} \hat{\mathbf{E}}_{n}^{*}
$$

${ }^{3}$ The matrix $\mathbf{A}(\boldsymbol{\tau})$ will have full rank if and only if its columns $\mathbf{a}_{1}\left(\tau_{1}\right), \mathbf{a}_{2}\left(\tau_{2}\right), \ldots, \mathbf{a}_{K}\left(\tau_{K}\right)$ are linearly independent for all possible realizations of $\boldsymbol{\tau}$. This is desirable and most likely the case for a DSCDMA system in which the orthogonality of different users' signature waveforms is maintained to a maximum extend to keep their mutual interference sufficiently low. The matrix $\mathbf{P}$ has full rank if $P_{k}>0$ for all $k$, which is obviously the case, since we assume all the users are active.

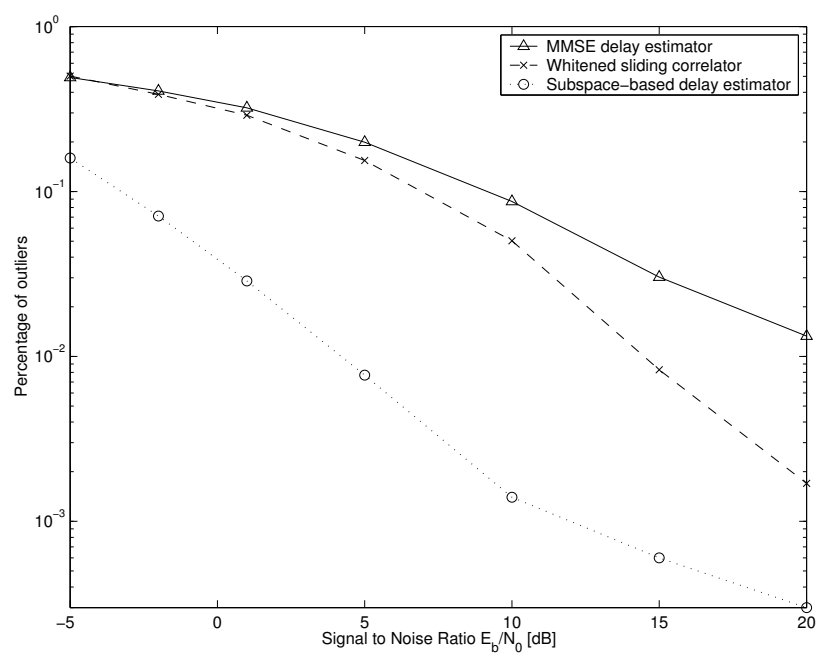

Figure 3: Acquisition error probability as a function of SNR.

where the columns of $\hat{\mathbf{E}}_{n}$ are the eigenvectors corresponding to the $N-K$ smallest eigenvalues of $\hat{\mathbf{R}}$. Note, however, that the columns of $\mathbf{A}$ will now be only approximately orthogonal to the columns of $\hat{\mathbf{E}}_{n}$. The above idea leads to the subspace-based delay estimator formulated as follows

$$
\hat{\tau}_{k}^{s}=\arg \min _{\tau_{k} \in[0, T)} J_{k, S}\left(\tau_{k}\right)=\arg \min _{\tau_{k} \in[0, T)} \frac{\left\|\hat{\mathbf{E}}_{n}^{*} \mathbf{a}_{k}\left(\tau_{k}\right)\right\|^{2}}{\left\|\mathbf{a}_{k}\left(\tau_{k}\right)\right\|^{2}}
$$

$J_{k, S}$ can be written analogously to (13) in order to estimate the delay analytically

$J_{k, S}\left(\tau_{k}\right)=\frac{\left(K_{1}+K_{2}-2 K_{3}\right) \delta^{2}+\left(2 K_{3}-2 K_{1}\right) \delta+K_{1}}{\left(2 N-2 K_{4}\right) \delta^{2}+\left(4 K_{4}-2 N\right) \delta+N}$

where $K_{1}=\left\|\hat{\mathbf{E}}_{n}^{*} \mathbf{a}_{k}\left(t_{0}\right)\right\|^{2}, K_{2}=\left\|\hat{\mathbf{E}}_{n}^{*} \mathbf{a}_{k}\left(t_{1}\right)\right\|^{2}, K_{3}=$ $\operatorname{Re}\left\{\mathbf{a}_{k}^{*}\left(t_{0}\right) \hat{\mathbf{E}}_{n} \hat{\mathbf{E}}_{n}^{*} \mathbf{a}_{k}\left(t_{1}\right)\right\}, K_{4}=\mathbf{a}_{k}\left(t_{0}\right)^{*} \mathbf{a}_{k}\left(t_{1}\right)$.

Figure 2 shows this subspace-based algorithm outperforms the WSC and MMSE estimator. The performance of all the algorithms is improved with longer training sequence. Figure 3 shows the SNR performance of these acquisition algorithms. As expected, strong noise deteriorates the performance of all the delay estimators. A reasonable level of signal to noise ratio has to be maintained for the algorithms to work.

\subsection{APPROXIMATE MAXIMUM LIKELIHOOD (AML) DELAY ESTIMATOR}

The problem with the estimators presented above is the slow convergence (large overhead), they all need a long training sequence to combat noise and fading and get an accurate estimate of the covariance matrix. The AML estimator is proposed to solve this problem. The complexity of ML estimation can be significantly reduced by some 
approximation methods, similar to the ideas of successive interference cancellation (SIC) and parallel interference cancellation (PIC) in data demodulation. These algorithms requires much less pilot symbols, thus achieve much faster convergence compared to the previously introduced schemes.

\subsubsection{Successive ML delay estimator}

In [9], a hierarchic way to construct an ML approximation for delay estimation was proposed. A revised version of this algorithm adopted to the system model in question is as follows:

1. Evaluate $J\left(\tau_{k}\right)=\operatorname{trace}\left(\mathbf{P}_{\mathbf{a}_{k}}^{\perp} \hat{\mathbf{R}}\right)$ for $k=$ $1,2, \ldots, K$. Choose $\mathbf{a}_{n_{1}}\left(\hat{\tau}_{n_{1}}\right)$ corresponding to the minimum trace value, which means we fix the delay estimate $\hat{\tau}_{n_{1}}$ for the user $n_{1}$.

2. Form the matrices $\mathbf{A}_{k}=\left[\mathbf{a}_{k}\left(\tau_{k}\right) \mathbf{a}_{n_{1}}\left(\hat{\tau}_{n_{1}}\right)\right]$ for $k=$ $1,2, \ldots, K$ and $k \neq n_{1}$. Compute $\mathbf{P}_{\mathbf{A}_{k}}^{\perp}=\mathbf{I}_{N}-\mathbf{A}_{k} \mathbf{A}_{k}^{\dagger}$, evaluate $J\left(\tau_{k}\right)=\operatorname{trace}\left(\mathbf{P}_{\mathbf{A}_{k}}^{\perp} \hat{\mathbf{R}}\right)$ for $k=1,2, \ldots, K$ and $k \neq n_{1}$. Choose $\mathbf{a}_{n_{2}}\left(\hat{\tau}_{n_{2}}\right)$ corresponding to the minimum trace value.

3. Form the matrices $\mathbf{A}_{k}=\left[\mathbf{a}_{k}\left(\tau_{k}\right) \mathbf{a}_{n_{1}}\left(\hat{\tau}_{n_{1}}\right) \mathbf{a}_{n_{2}}\left(\hat{\tau}_{n_{2}}\right)\right]$ for $k=1,2, \ldots, K$ and $k \neq n_{1}, n_{2}$. Compute $\mathbf{P}_{\mathbf{A}_{k}}^{\perp}=$ $\mathbf{I}_{N}-\mathbf{A}_{k} \mathbf{A}_{k}^{\dagger}$, evaluate $J\left(\tau_{k}\right)=\operatorname{trace}\left(\mathbf{P}_{\mathbf{A}_{k}}^{\perp} \hat{\mathbf{R}}\right)$. Choose $\mathbf{a}_{n_{3}}\left(\hat{\tau}_{n_{3}}\right)$ corresponding to the minimum trace value.

4. Repeat step 3 until all the users' delays are estimated and fixed.

The idea of this scheme is similar to that of the SIC. We fix the delay of one user at each step, that user's signal is reconstructed and used for detecting the next user's delay.

\subsubsection{Parallel ML delay estimator}

The complexity of ML algorithm can also be reduced in an iterative multistage manner like PIC. We use the conventional delay estimator (SC) to get an initial estimates of delays for all the users and enter the iteration loop. The subsequent stages differ from the successive estimator introduced earlier. Instead of fixing one user's delay at a time, we fix the delays of all the interfering users simultaneously (in parallel), using the estimates derived from last iteration in order to estimate the delay for the user of interest, e.g., the $k^{t h}$ user. This multistage parallel ML delay estimator can be expressed as

$$
\begin{aligned}
\hat{\boldsymbol{\tau}}_{k}^{(l)} & =\left.\arg \min _{\tau_{k}} \sum_{j=1}^{L}\left\|\mathbf{r}(j)-\hat{\mathbf{A}}^{(l-1)}\left(j, \tau_{k}\right) \mathbf{h}(j)\right\|^{2}\right|_{\mathbf{h}(j)=\hat{\mathbf{A}}^{\dagger} \mathbf{r}(j)} \\
& =\arg \min _{\tau_{k}} \operatorname{trace}\left(\mathbf{P}_{\hat{\mathbf{A}}}^{\perp} \hat{\mathbf{R}}\right)
\end{aligned}
$$

The matrix $\hat{\mathbf{A}}^{(l-1)}\left(\tau_{k}\right)$ is defined as $\hat{\mathbf{A}}^{(l-1)}\left(j, \tau_{k}\right)=\left[\mathbf{a}_{1}\left(j, \hat{\tau}_{1}^{(l-1)}\right) \cdots \mathbf{a}_{k}\left(j, \tau_{k}\right) \cdots \hat{\mathbf{a}}_{K}\left(j, \hat{\tau}_{K}^{(l-1)}\right)\right]$

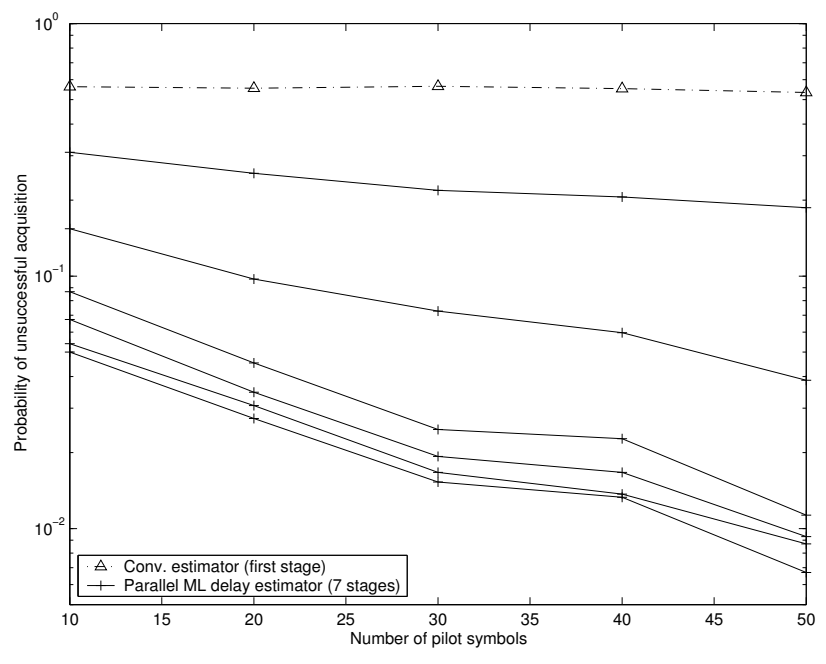

Figure 4: Acquisition performance of the multistage parallel $M L$ delay estimator.

where $\hat{\tau}_{1}^{(l-1)}, \cdots, \hat{\tau}_{K}^{(l-1)}$ are the estimated delays for the interfering user $1, \cdots, K$ at the $(l-1)^{t h}$ iteration stage.

In contrast to the original ML algorithm which jointly detects all the users, these two approximate ML algorithms detect the delay of one user at a time, thus greatly reduce the computational complexity. Like the SC, they have the property of fast convergence, require much shorter training sequence than the WSC, MMSE and subspace-based algorithms, thus significantly reduce the overhead induced by the training.

The results of the 7-stage parallel ML delay estimation are demonstrated in figure 4. Clearly, the performance is improved at each iteration. However, it tends to get saturated at the $7^{\text {th }}$ stage.

The comparison among different estimators is illustrated in figure 5. The parallel ML estimator performs slightly better than the successive one. They both achieve good acquisition performance with a relatively short training sequence. As expected, the subspace-based estimator does not work with short training sequences. Its performance improves considerably as the number of the pilots increases and eventually becomes better than the AML estimators when more than 250 pilots are used.

\section{Conclusions}

In this paper, several pilot-assisted multi-user acquisition algorithms, namely the WSC, MMSE, subspacebased, and AML estimators for estimating propagation delays of orthogonally modulated signals over Rayleighfading channels are introduced. They reduce the computational complexity of the ML estimation from exponential to polynomial, which makes them more feasible for implementation. Their performance is also evaluated and compared in this paper. Simulation results show that they 


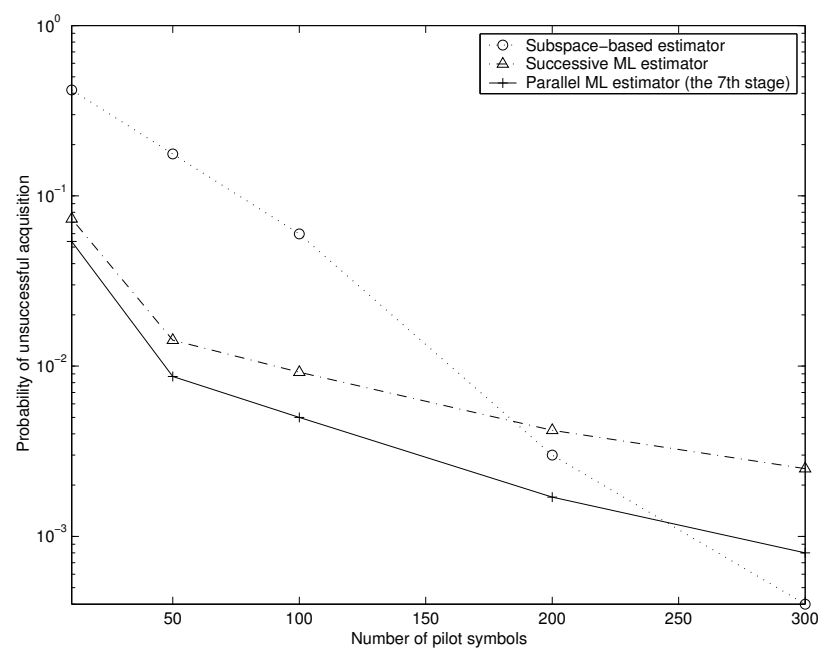

Figure 5: Comparison between subspace-based estimator and AML estimators.

achieve good acquisition performance in presence of the MAI. This is in contrast to the conventional SC which only works in single user environment.

By comparison, the subspace-based algorithm in general gives more reliable and more accurate estimate of delays at the expense of large overhead induced by the training. While the overhead is greatly reduced by the iterative AML algorithms with increased computational complexity which is needed for iteration process. If the receiver has fast and powerful signal processing capability, which is the case in the base station, the AML algorithms are preferred.

All the algorithms introduced in this paper require users' training at the same time, they are therefore applicable when we have a dedicated pilot channel for synchronization. This should be feasible because in some $3 \mathrm{G}$ standard, the I-channel is employed for transmitting data, while Q-channel is the control channel reserved for synchronization and channel estimation.

\section{REFERENCES}

[1] P. Xiao, E. Ström. Performance of iterative DS-CDMA M-ary demodulation in the presence of synchronization errors. In Vehicular Technology Conference, pages 17031707, May, 2001.

[2] E. Ström, F. Malmsten. A maximum likelihood approach for estimating DS-CDMA multipath fading channels. IEEE Journal on Selected Areas in Communications, vol. 18, pages 132-140, Jan. 2000.

[3] D. Zheng, J. Li, S. Miller, E. Ström. An efficient codetiming estimator for DS-CDMA signals. IEEE Trans. Signal Processing, vol. 45, pages 82-89, Jan. 1997.

[4] E. Ström, S. Parkvall, S. Miller, B. Ottersten. Propagation delay estimation in asynchronous direct-sequence codedivision multiple access systems. IEEE Trans. Commun., vol. 44, pages 84-93, Jan. 1996.
[5] T. Ojanperä and R. Prasad. An overview of air interface multiple access for IMT-2000/UMTS. IEEE Communications Magazine, vol. 36, pages 82-95, Sept. 1998.

[6] P. Xiao, E. Ström. Synchronization algorithms for iteratively demodulated M-ary DS-CDMA systems. In Global Telecommunications Conference, pages 1371-1375, Nov. 2001.

[7] E. Ström, S. Miller. Iterative demodulation of orthogonal signalling formats for DS-CDMA. In International Conference on Communications, vol. 3, pages 1457-1461, 2000.

[8] S. Bensley, B. Aazhang. Subspace-based estimation of multipath channel parameters for CDMA communication systems. In Global Telecommunications Conference, pages 154-157, 1994.

[9] J. Joutsensalo, J. Lilleberg, A. Hottinen, J. Karkunen. A hiearchic maximum likelihood method for delay estimation in CDMA. In Vehicular Technology Conference, vol. 1, pages 188-192, 1996.

[10] P. Xiao, E. Ström. Acquisition of orthogonal modulated signals in Rayleigh-fading channels. In European Wireless, pages 811-816, Feb. 2002.

[11] P. Xiao, E. Ström. Applications of maximum likelihood algorithm in asynchronous CDMA systems. To appear in International Symposium on Spread Spectrum Techniques and Applications, Sept. 2002. 